\title{
Robust specular reflection removal and visibility enhancement of endoscopic images using 3-channel thresholding technique and image inpainting
}

\author{
Wooju Alex Lim \\ Deerfield Academy, US \\ wujulim@gmail.com
}

\begin{abstract}
Specular reflections create artifacts in endoscopic images, which may lead to misdiagnosis. In this paper, we propose a method for robust removal of specular reflections by using a thresholding technique in each of the RGB channels to segment the specular reflections from images. We further use dilation to ensure full local segmentation and inpainting to replace the areas of reflections with non-specular regions. Our method also provides a visibility enhancement feature to improve the decreased brightness due to the reflection removal by using the gamma-correction, histogram shift, and histogram equalization. On the Iparkmall Clinic dataset, our method has achieved average Peak-to-Signal-Noise Ratio (PSNR) of $42.62 \mathrm{~dB}$ with a standard deviation of $5.80 \mathrm{~dB}$ and a minimum value of $23.3 \mathrm{~dB}$. The average processing time was $219 \mathrm{~ms}$, enabling average 45 frames per second (FPS) processing speed on an Intel i7 processor.
\end{abstract}

Keywords. Specular Reflections, endoscopic images, 3-channel thresholding technique, image inpainting

\section{INTRODUCTION}

Endoscopy is a gold-standard imaging modality for gastrointestinal cancer screening. During an endoscopy, a flexible tube with a camera and a light source is inserted into the patient's body, which enables the clinician to visually analyze the internal organs. In addition to human diagnosis, computeraided diagnosis using endoscopic im- ages, such as the automatic polyp detection, has recently become an area of current interest. However, computer- aided approaches suffer from specular reflections in the endoscopic images Fig. 1, which arise from light reflecting from the wet surface of the mucous membrane [1].

Due to the bright white spots from the specular reflections, valuable visual information contained in the image is occluded. In particular, flat polyps are often unrecognized or have their size miscalculated due to the specular reflections, despite the potential for development into a malignancy. This phenomenon leads to a lower chance of early-stage diagnosis and treatment of cancer [2]. There- fore, there is a clear need for suppressing specular reflections in order to enable accurate human and computer- aided diagnosis. In this paper, we propose an adaptive method of threshold filtering to segment the specular re- flections and remove them by image inpainting. 


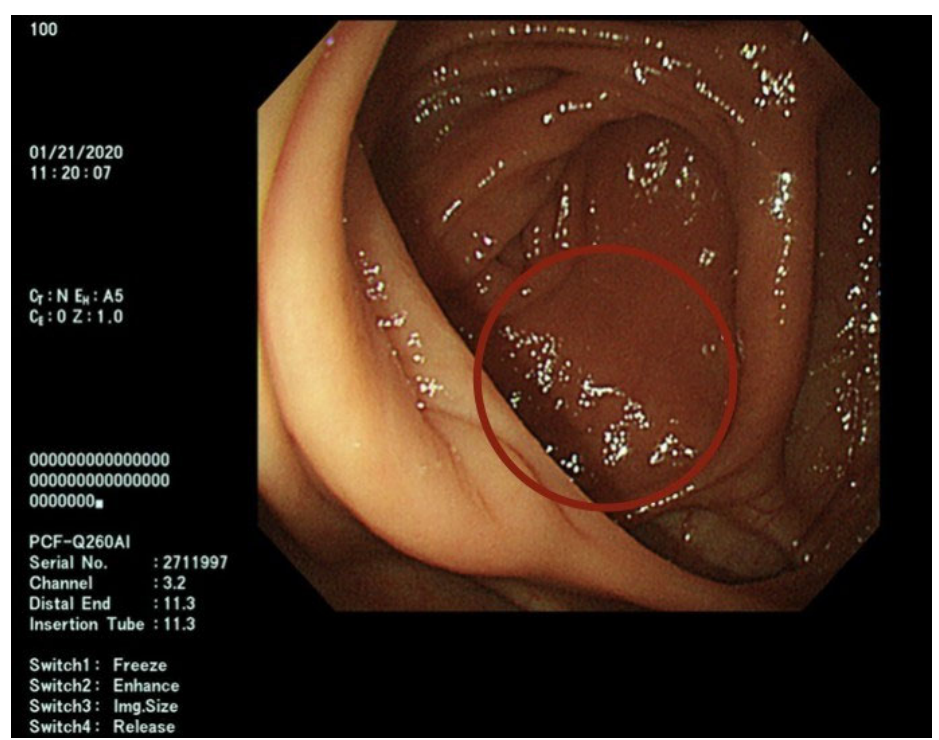

FIG. 1. Specular reflections in endoscopic images

\section{RELATED WORK}

Previous methods for specular reflection removal include various image processing algorithms and conversions to different color spaces. Oh et al.[3] proposed a method to convert RGB endoscopic images into HSV color space and detect specular reflection by thresholding the $\mathrm{S}$ and V values. However, this method had varying performance over different illumination conditions and also color space conversion is a computationally-intensive procedure. Meslouhi et al. [4] proposed a method to create a copy of the RGB endoscopic image, convert it into a CIE-XYZ color space, and segment the specular reflection region by calculating the scalar luminance difference between the two color spaces, using the latter as a threshold. This method then creates a pyramidal image to allow multi-scale reconstruction of the specular reflection regions, and then performed histogram equalization to restore the brightness. Through this procedure, this method achieved a semi-realistic reconstruction of specular reflected regions. However, it also involved high computational complexity and memory requirements. Ghosh et al. [2] proposed a method to apply thresholding adaptively in the individual color channels to select the candidates for specular reflected regions, and then further narrowed down the selection by applying a median filter and then a threshold on the non-specular regions. De- spite good accuracy of the adaptive thresholding in the individual color channels, this method has only been successful in colonoscopy.

In the approach proposed here, we avoid the color space conversion that was used by Oh et al. [3], and segment the specular reflected regions using individual color thresholding following Ghosh et al. [2], and then enhance the image quality by histogram equalization as used in Meslouhi et al [4].

\section{METHODOLOGY}

\subsection{Three-channel thresholding}

The typical general-purpose thresholding methods process grayscale images, which are obtained in pre- processing by converting from a color to grey-scale encoding [5]. However, endoscopic images generally contain bright regions of different colors, as well as specular reflections. To successfully segment specular reflections from the regions that are bright but not specularly-reflecting, modifications to the typical thresholding technique are necessary. Instead of processing a single grayscale image, the proposed method is based on extracting each color channel from the 3-color image (RGB). Then each channel is individually processed by a thresholding algorithm, and the reflections commonly detected by all three channels are used for the final seg- mentation. Eq. (1) describes the thresholding algorithm. 


$$
I(x, y)\left\{\begin{array}{l}
0, \text { if } I_{R}(x, y)>T \cap \quad I_{G}(x, y)>T \cap I_{B}(x, y)>T \\
I(x, y), \text { otherwise, }
\end{array}\right.
$$

where $I_{R}, I_{G}, I_{B}$ is pixel intensity in each red, green, blue channel of image, $(x, y)$ is the location of the pixel in the image, and $T$ is the threshold value. The processed image is shown in Fig. 2.

\subsection{Dilation}

From the dataset, it was observed that chromatic im- age aberration occurred in specular reflections. An ex- ample of this phenomenon is shown in Fig. 3.

The aberrations were composed of white pixels in the center and shades of different colors surrounding the for- mer. The proposed thresholding technique is calibrated to exclusively detect the white pixels. Therefore, the segmented specular reflections appeared smaller than what is seen on the color image, which would cause inaccurate interpolation, once the inpainting algorithm is applied. An image dilation algorithm with a kernel convolution $5 \times 5$ matrix was used to expand the segmented area un- til the entire local specular reflection is covered. Eq. (2) describes the convolution process.

$$
\begin{aligned}
g(x, y) & =\omega * f(x, y) \\
& =\sum_{d x=-2}^{2} \sum_{d y=-2}^{2} \omega(d x, d y) f(x+d x, y+d y)
\end{aligned}
$$
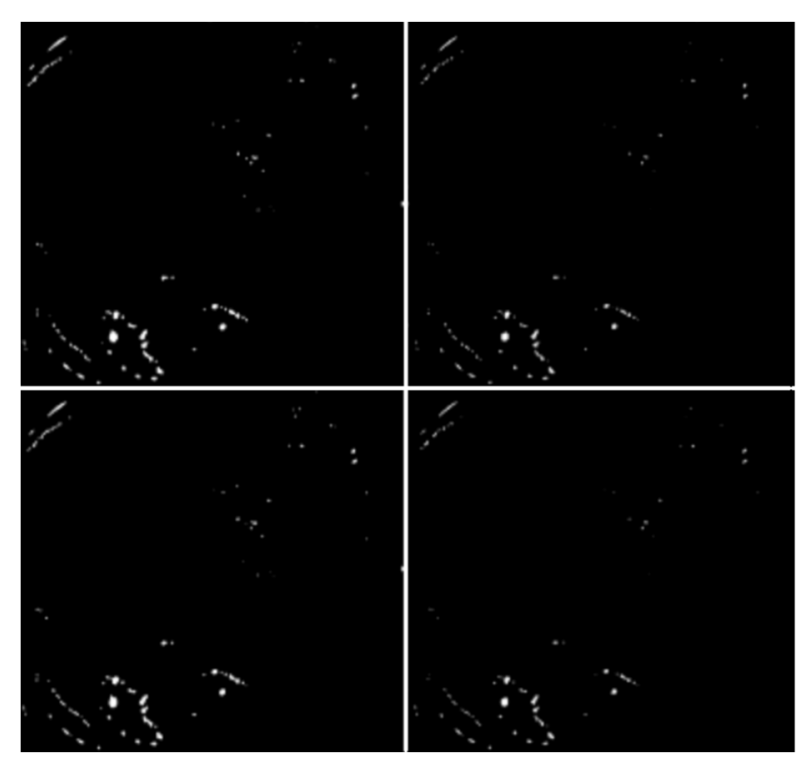

FIG. 2. Image thresholding applied to red, green blue channel, and the intersection image of the three channels

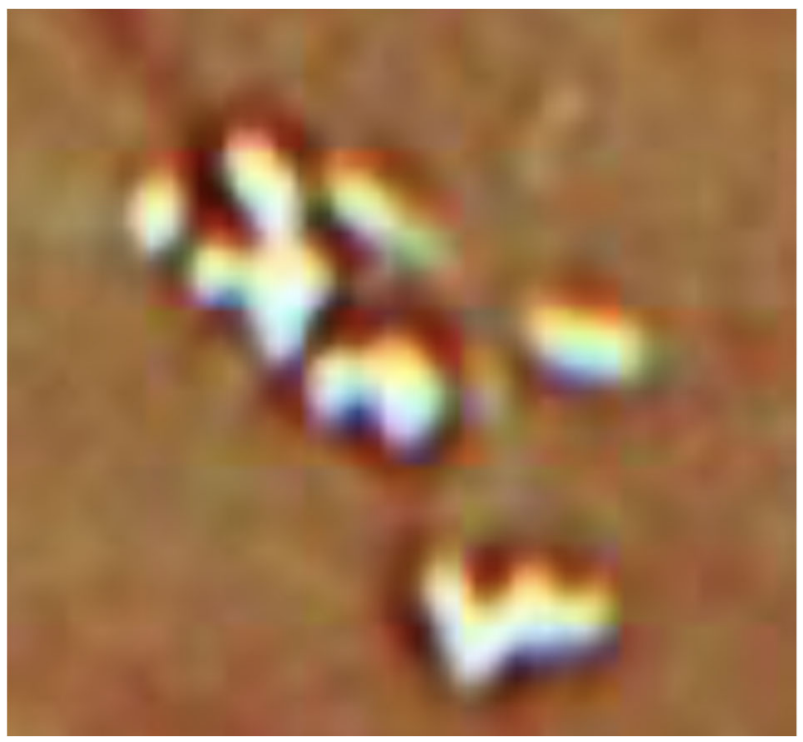

FIG. 3. Example of chromatic aberration in endoscopic image

, where $g(x, y)$ describes the pixel location in the out- put image, $\omega$ describes the kernel, $f(x, y)$ describes the pixel location in the input image, and $d x, d y$ describe kernel element multiplication.

In particular, an elliptical convolution kernel shown in Eq. (3), which is created from morphological transformation was used to preserve the curvature of the specular reflection regions. 


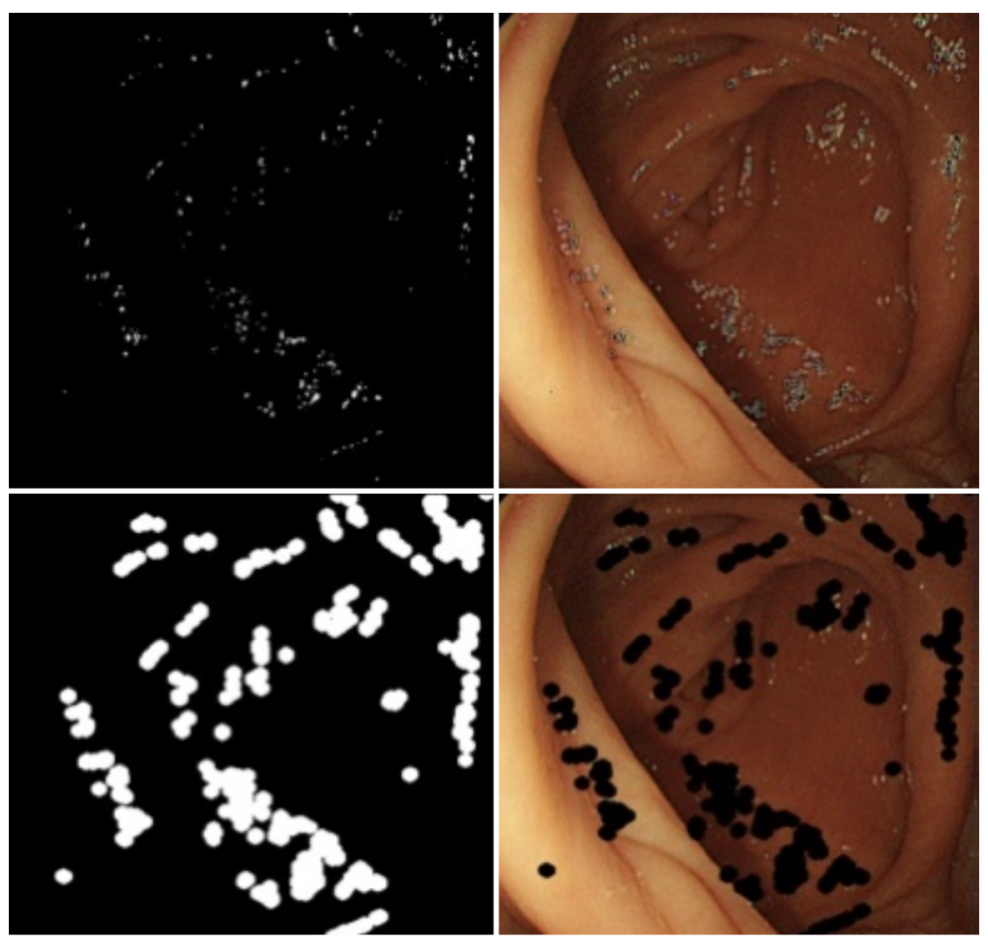

FIG. 4. Specular reflection remains in the image even after thresholding, due to chromatic aberration Dilating the masked region successfully removed all specular reflection.

$$
\omega=\left[\begin{array}{lllll}
0 & 0 & 1 & 0 & 0 \\
1 & 1 & 1 & 1 & 1 \\
1 & 1 & 1 & 1 & 1 \\
1 & 1 & 1 & 1 & 1 \\
0 & 0 & 1 & 0 & 0
\end{array}\right]
$$

The dilated region was then considered as mask and removed from the original image. The result is as shown in Fig. 4.

\subsection{Inpainting}

The inpainting algorithm filled the dilated regions with non-specular representations, interpolating from the neighboring pixels [6]. Eq. (4) shows that, for an empty pixel $p$, the algorithm calculates the first-order approximation of the gradient value from close neighboring pixels, as shown in Fig. 5. Using the first order- approximation of the gradient value, the intensity of the empty pixel $p$ can be estimated, using linear interpolation.

$$
I(p)=\frac{\sum_{q \in B_{\varepsilon}(p)} w(p, q)[I(q)+\nabla I(q)(p-q)]}{\sum_{q \in B_{\varepsilon}(p)} w(p, q)}
$$

, where $T$ is the interpolated pixel intensity value, $(p, q)$ is the pixel location, $\nabla I(q)$ is the image gradient at pixel $q$, and $w$ is the weighting function.

These gradient values were then weighted by the normalized weighting function, which is based on Gaussian distribution function, which is described in Eq. (5). 

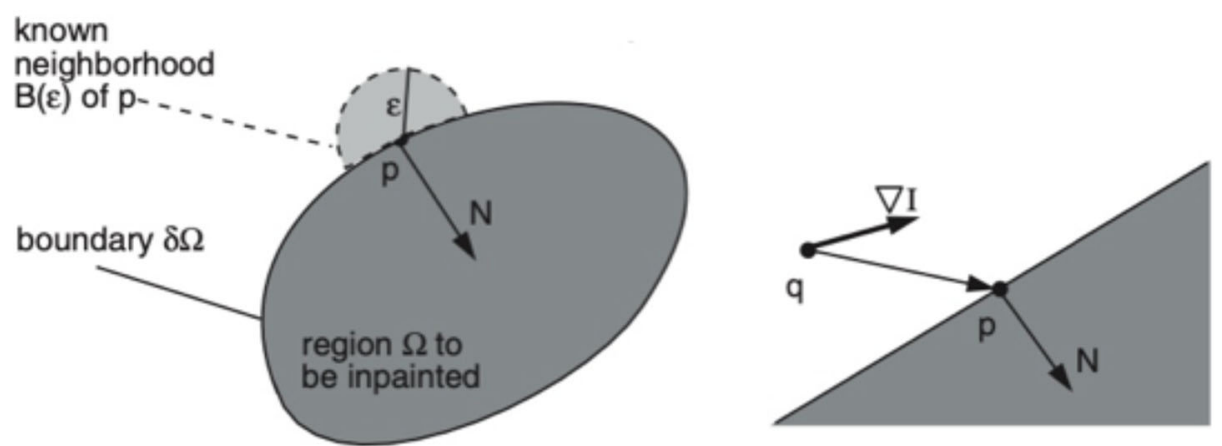

FIG. 5. The inpainting algorithm from Telea [6]

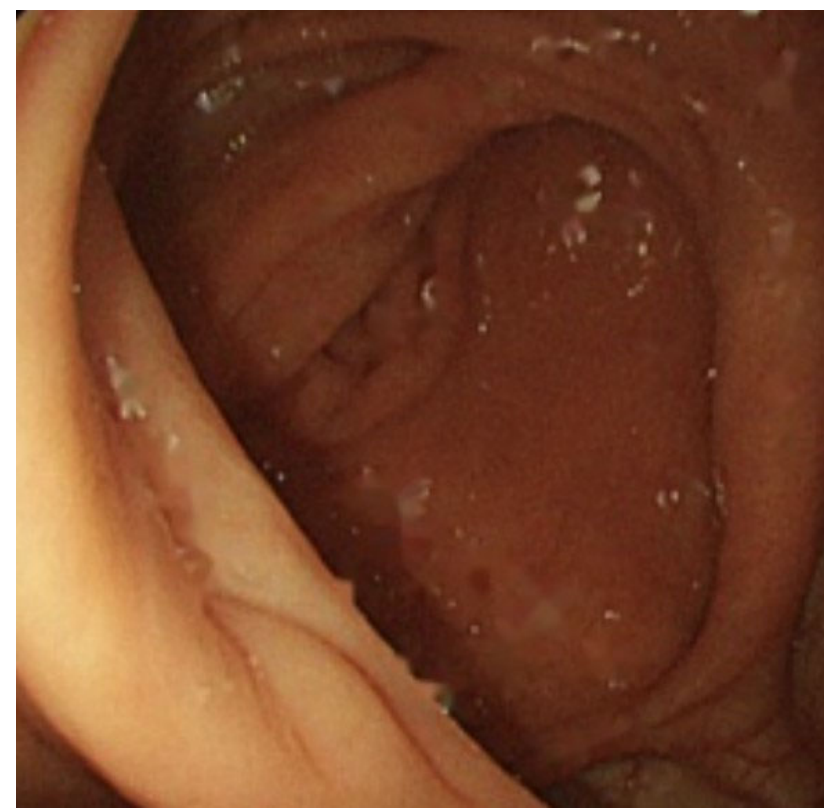

FIG. 6. Inpainted image after specular reflection removal

$$
\mathcal{N}(x ; \mu, \sigma)=\frac{1}{\sqrt{2 \pi \sigma^{2}}} \exp \left[-\frac{1}{2}(x-\mu)^{2} / \sigma^{2}\right]
$$

, where $\mathcal{N}(\mathrm{x} ; \mu ; \sigma)$ is the normal distribution with mean value $\mu$ and standard deviation $\sigma$.

This method therefore reconstructed a semi-realistic representation of non-specular tissue in the image, as shown in Fig 6.

\subsection{Visibility Enhancement}

Despite the successful reconstruction of semi-realistic non-specular tissue in place of the specular reflections, the entire image becomes slightly darker on average since the bright regions in the image are removed. Therefore, to enhance visibility, gamma-correction was applied to increase the brightness of the dark regions. However, using gamma-correction unavoidably brightened the entire image. Therefore histogram shift and equalization were applied to maintain the distribution of the dark and bright regions over the entire image, as shown in Fig 7. 


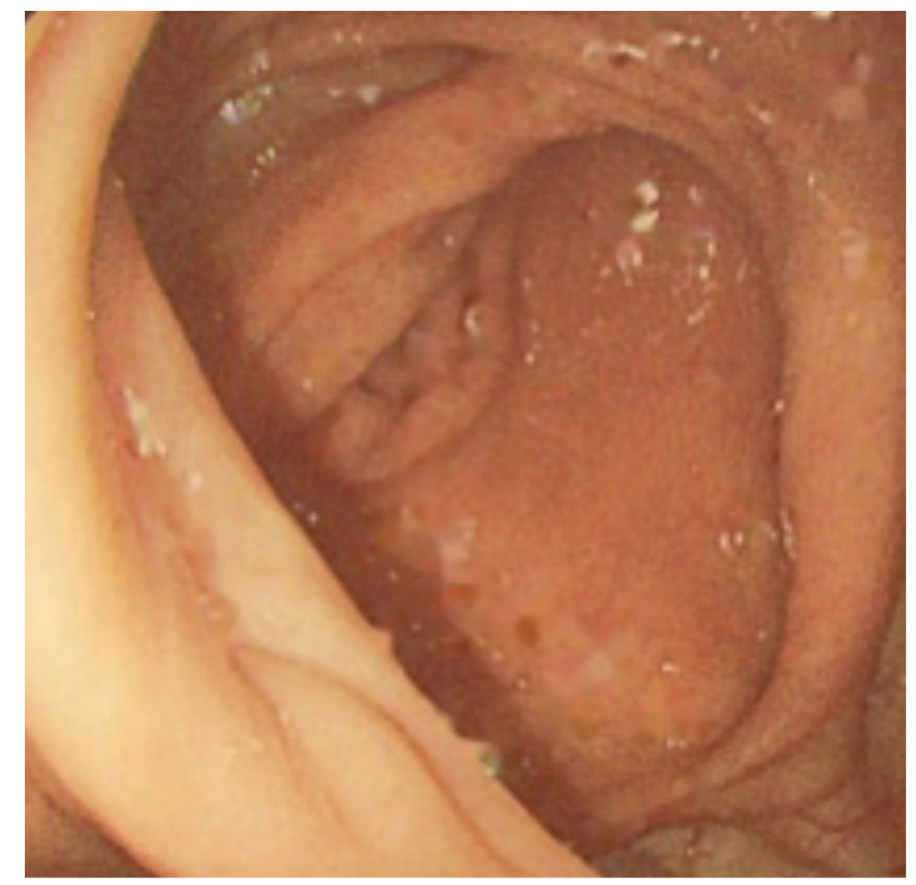

FIG. 7. Endoscopic image after visibility enhancement using histogram shift and equalization
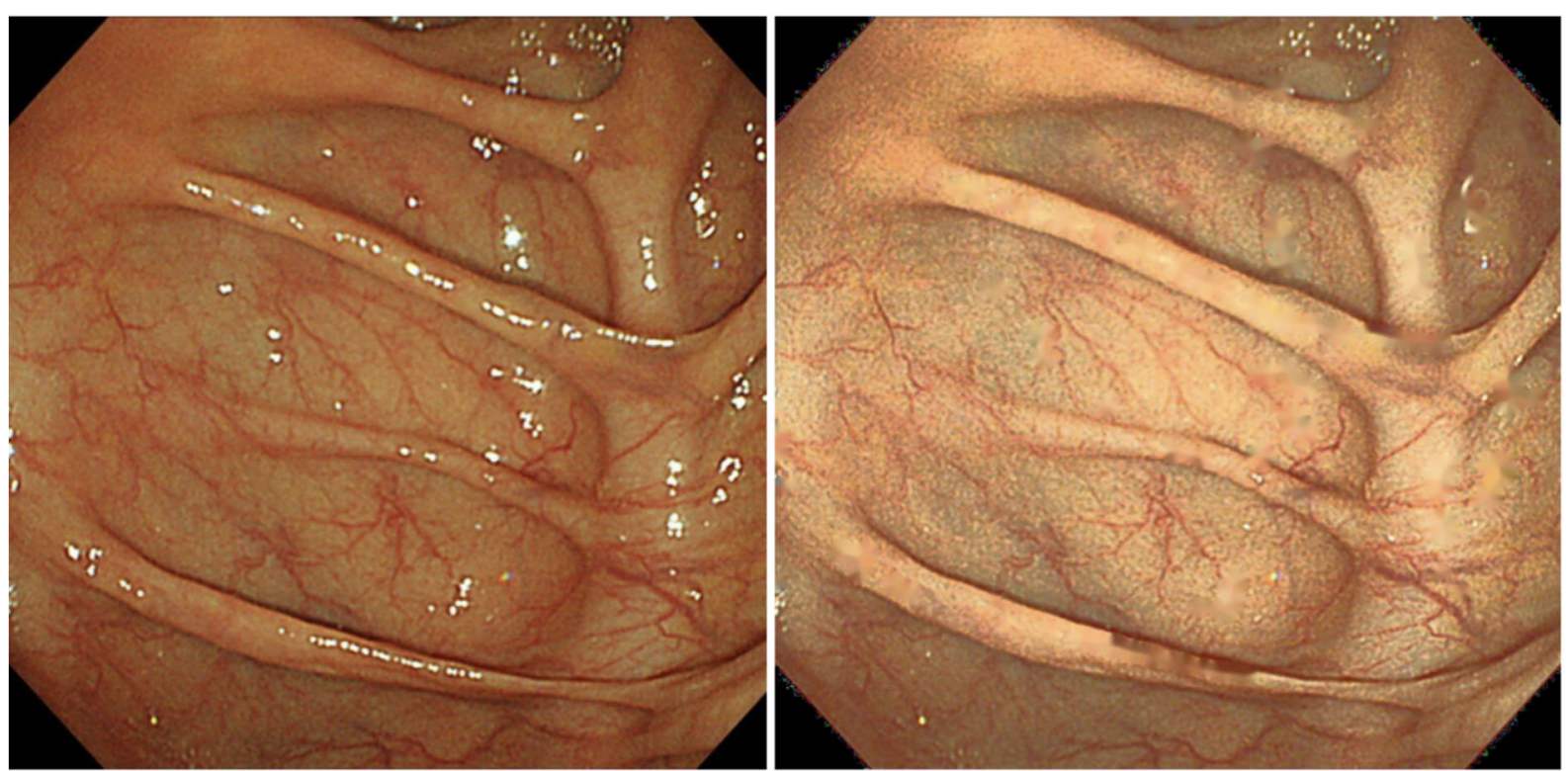

FIG. 8. Comparison of raw input image on the left and the final processed image on the right

Fig. 8 shows the raw image on the left and the enhanced image on the right.

\section{QUANTITATIVE ANALYSIS}

For the numerical experiment, a data set of 375 endoscopic images acquired by Iparkmall Clinic (Seoul, South Korea) was used. The data set has been extracted from the archive that documents images from regular health screenings. All patients of the endoscopic images have been notified and provided consent to data usage for research. The endoscopic images were captured with an Olympus endoscope. As shown in Fig. 1, the original images included black letterboxes with metadata. Since these areas of the images are not used in the algorithm, they were removed prior to processing. For all 
the algorithms discussed above, an open-sourced image processing and computer vision software library, called OpenCV (version 4.3.0.) was used.

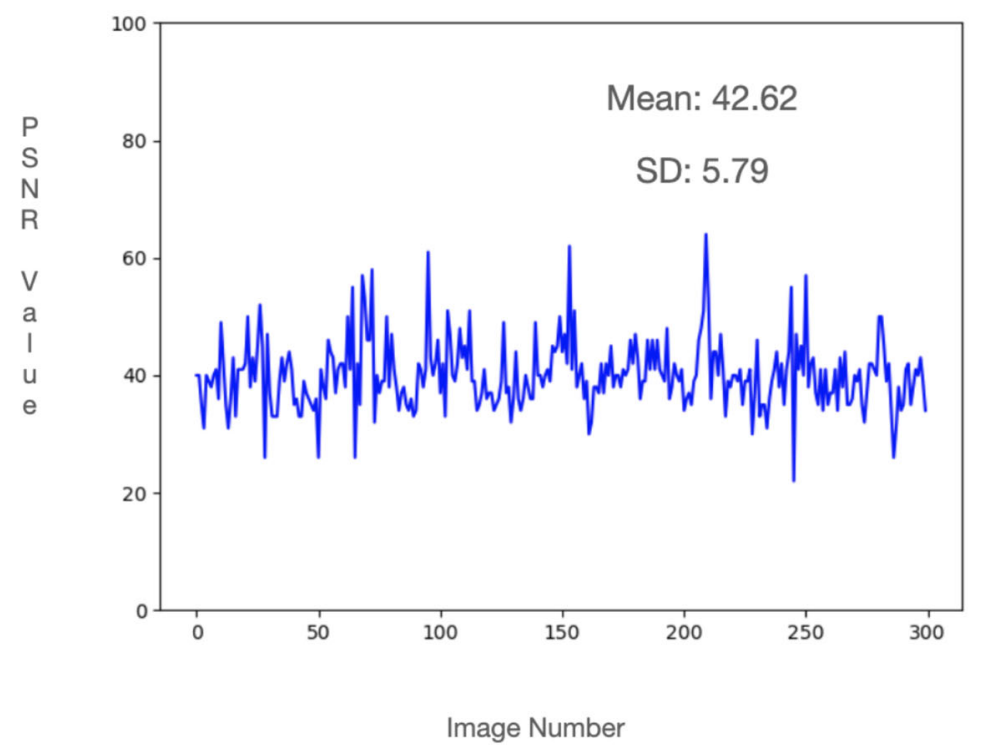

FIG. 9. PSNR values over 375 images in Iparkmall dataset

To validate the performance of the proposed method on realistic endoscopic images, we performed a quantitative analysis. Due to the nature of the data set, there was no gold standard image available. Therefore, gold standard images were heuristically created. The gold standard image was created by removing the specular reflections, through threshold segmentation and inpainting. Then, the specular reflections of another image chosen from the dataset were segmented and masked on gold standard image. The masked gold standard image was processed through the proposed method to remove the artifacts. Based on these images, peak signal to noise ratio (PSNR) values were calculated and used as an accuracy metric for this method, as defined in (6). For the PSNR metric, higher values indicate better performance, as they represent the similarity of the gold standard image and the final processed image.

$$
\begin{aligned}
P S N R & =10 \cdot \log _{10}\left(\frac{M A X_{I}^{2}}{M S E}\right) \\
& =20 \cdot \log _{10}\left(\frac{M A X_{I}}{\sqrt{M S E}}\right) \\
& =20 \cdot \log _{10}\left(M A X_{I}\right)-10 \cdot \log _{10}(M S E)
\end{aligned}
$$

All 375 images were processed by the proposed method, and the average PSNR value was $42.62 d B$ with the standard deviation value of $5.80 \mathrm{~dB}$. The histogram of the PSNR population is shown in Fig. 9.

Other existing specular reflection suppression methods, although tested on different data sets, have assessed their methods to be successful with an average PSNR value of 30.74 [7], 31.24 [8], 29.39 [9]. Our method has achieved $42.62 \mathrm{~dB}$ throughout the data set with he minimum value $23.3 \mathrm{~dB}$. We conclude that the pro- posed method has performed successfully on the Ipark- mall Clinic data set. 
Also, for an Intel i7 processor with the $3.0 \mathrm{GHz}$ clock speed, we achieved the average processing time of $219 \mathrm{~ms}$, which is roughly $4-5$ frames per second.

\section{DISCUSSION AND CONCLUSIONS}

In this paper, a specular reflection suppression method was developed based on an adaptive 3-channel thresholding technique, dilation, and inpainting. Our results indicate that the proposed method not only located the specular reflections but also removed them successfully. The average PSNR value of $42.62 \mathrm{~dB}$ and the strong consistency of PSNR values for the population showed that the proposed method was robust.

The main limitation of this study is that, despite a good PSNR value that has been achieved, a direct com- parison with other methods has not been performed on the same data set. This is because the other methods' source codes have not been publicly released, so that a direct test under on the data set available to us could not be performed. However, considering that the Iparkmall Clinic data set has been acquired under typical conditions with various viewpoints, our method can be considered as robust to various illuminations and viewpoints.

We also note that, although a good PSNR values were obtained from the output of the algorithm, structural discontinuities were frequently observed in the recon- structed region and the original image. This is mainly due to the limitation of the inpainting algorithm that only calculates a first-order approximation of the pixel values. Also, the low quality of the gold-standard images could have caused this issue.

The possible future avenues of research include applying machine learning techniques to non-linear filters on the three-dimensional color space and investigating the characteristic locations of the specular reflections in the three-dimensional color space. This might increase the accuracy of speckle suppression.

\section{REFERENCES}

[1] Akbari M, Mohrekesh M, Najariani K, Karimi N, Samavi S, Soroushmehr SMR. Adaptive specular reflection de- tection and inpainting in colonoscopy video frames. Proceedings - International Conference on Image Processing, ICIP. 2018;p. 3134-3138.

[2] Ghosh A, Arnold M, Ameling S, Lacey G. Automatic segmentation and inpainting of specular highlights for endoscopic imaging. Eurasip Journal on Image and Video Processing. 2010;2010.

[3] Oh JH, Hwang S, Lee JK, Tavanapong W, Wong J, de Groen PC. Informative frame classification for endoscopy video. Medical Image Analysis. 2007;11(2):110- 127.

[4] Meslouhi OE, Kardouchi M, Allali H, Gadi T, Benkaddour YA. Automatic detection and inpainting of specular re- flections for colposcopic images. Open Computer Science. 2011;1(3):341354.

[5] Shafait F, Keysers D, Breuel TM. Efficient Implementation of Local Adaptive Thresholding techniques using integral images. Document Recognition and Retrieval XV. 2008;6815:681510681510 .

[6] Telea A. An Image Inpainting Technique Based on the Fast Marching Method. Journal of Graphics Tools. 2004;9(1):23-34.

[7] Criminisi A, Pérez P, Toyama K. Region filling and object removal by exemplar-based image inpainting. IEEE Transactions on Image Processing. 2004;13(9):1200-1212.

[8] Anupam, Goyal P, Diwakar S. Fast and enhanced algorithm for exemplar based image inpainting. Proceedings

- 4th Pacific-Rim Symposium on Image and Video Technology, PSIVT 2010. 2010;p. 325-330.

[9] Yin L, Chang C. An effective exemplar-based image in- painting method. International

Conference on Communication Technology Proceedings, ICCT. 2012;p. 739-743. 